\title{
EVOLUCIÓN DE LOS REPOSITORIOS DOCUMENTALES. EL CASO SOCIALNET
}

Pablo Lara-Navarra, Enric Serradell-López y David Maniega-Legarda

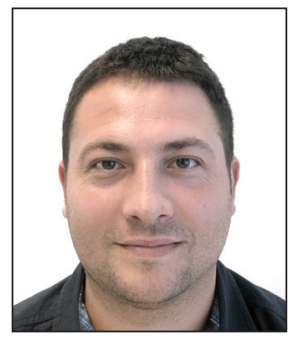

Pablo Lara-Navarra, es licenciado en documentación por la Universidad de Granada, doctor por la Universitat Pompeu Fabra, y master de Sociedad de la información y conocimiento por la Universitat Oberta de Catalunya (UOC). Su carrera se centra en la docencia, innovación en productos y consultoría a empresas. Es especialista en gestión de innovación, gestión de conocimiento y e-learning, temas sobre los que ha publicado numerosos libros y artículos. Ha sido director académico del Master de tecnologías accesibles UOC-Technosite y director de Innovación de la UOC. Es profesor de los Estudios de Ciencias de la Información y de la Comunicación de la UOC y asesor de empresas tecnológicas.

http://orcid.org/0000-0003-0595-3161

Universitat Oberta de Catalunya, Estudios de Información y Comunicación Rambla de Poblenou, 156. 08018 Barcelona, España plara@uoc.edu

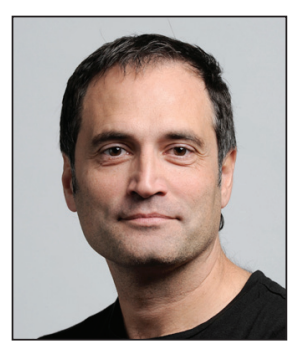

Enric Serradell-López es doctor en ciencias económicas y empresariales por la Universidad de Barcelona, profesor de la Universitat Oberta de Catalunya y director de programas MBA y directivos. Su docencia e investigación está vinculada al e-learning y al análisis de los intangibles como ventaja competitiva de las empresas. Es miembro de la Asociación Científica de Economía y Dirección de Empresas (Acede) y forma parte de la comisión de intangibles de la Asociación Catalana de Contabilidad y Dirección de Empresas (Accid). http://orcid.org/0000-0003-2719-1380

Universitat Oberta de Catalunya, Estudios de Economía y Empresa Av. Tibidabo, 39-32. 08035 Barcelona, España eserradell@uoc.edu

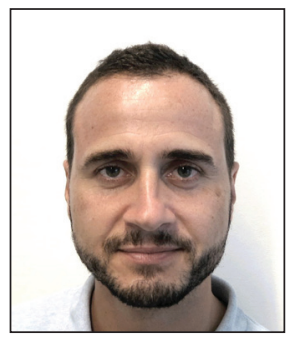

David Maniega-Legarda, historiador del arte y documentalista técnico, es fundador y chief technology officer (CTO) de ICAlia. Ha sido director de la Oficina Abierta de Innovación de la Universitat Oberta de Catalunya (UOC) y responsable del equipo web del Servicio de Cooperación Bibliotecaria del Depto. de Cultura y Medios de Comunicación de la Generalitat de Catalunya. Actúa como consultor de usabilidad y accesibilidad web, estrategia tecnológica y procesos de innovación en las organizaciones. Es experto en la creación y desarrollo de proyectos de innovación centrados en elearning. Es profesor en el Máster de documentación digital de la Universitat Pompeu Fabra donde además es coordinador de proyectos web.

http://orcid.org/0000-0002-9878-9446

ICAlia Solutions $S L$ Almogàvers, 119-123. 08018 Barcelona, España david.maniega@icalia.es

\section{Resumen}

Se describen algunos conceptos y tendencias tecnológicas tales como identidad digital (digital identity), grandes conjuntos de datos (big data), datos en abierto (open data) y visualización de datos (data visualization) que pueden aplicarse a los repositorios documentales. Se presenta el modelo de repositorio SocialNet, como ejemplo de proyecto innovador diseñado en consonancia con tales tendencias.

\section{Palabras clave}

Repositorios, Datos en abierto, Grandes datos, Gestión del conocimiento, Gestión de contenidos, Archivos abiertos, SocialNet.

\section{Title: Evolution of documents repositories. The SocialNet case}

\begin{abstract}
Some concepts and technology trends such as digital identity, large sets of data (big data) open data and data visualization that can be applied to document repositories are briefly described. In this article it is presented SocialNet repository model as an example of innovative project designed in line with such trends.
\end{abstract}




\section{Keywords}

Repositories, Open data, Big data, Knowledge management, Content management, Open archives, SocialNet.

Lara-Navarra, Pablo; Serradell-López, Enric; Maniega-Legarda, David. “Evolución de los repositorios documentales. El caso SocialNet". El profesional de la información, septiembre-octubre, v. 22, n. 5, pp. 432-439.

http://dx.doi.org/10.3145/epi.2013.sep.08

\section{Principales tendencias y retos en la gestión de información}

Para los profesionales de la información es de capital importancia monitorizar la evolución del mercado, analizando y conceptualizando las tendencias tecnológicas para tratar de anticipar el futuro. Tienen que detectar los cambios de comportamientos del consumidor, frente a los nuevos productos y servicios de información.

El mercado tecnológico de la información es analizado por grandes consultoras como Deloitte, Accenture, Gartner, $I D C$..., las cuales detectan problemas, necesidades y oportunidades en la gestión de información y realizan o encargan nuevos productos/soluciones para resolverlos. Concretamente Deloitte (2012) vaticina:

- cambio radical de entender los productos y servicios de información debido al auge de los smartphones y tablets, de los que la consultora estima que antes de 2016 se habrán vendido unos 1.600 millones de unidades;

- crecimiento vertiginoso en el uso de redes sociales, con unos 1.500 millones de usuarios;

- computación en la nube (cloud computing);

- tecnologías M2M (machine to machine).

Existe un consenso generalizado en cuanto a las innovaciones que pueden cambiar los modelos de información actuales. Entre ellas:

- Iudificación (gamification);

- computación en la nube (cloud computing);

- identidad digital (digital identity);

- grandes datos (big data);

- davi (data visualization),

- redes de colaboración/relación profesional (social enterprise), entre otras.

En este trabajo nos centraremos en identidad digital, grandes datos y visualización de datos, que consideramos determinarán la evolución de los repositorios documentales.

\section{Los repositorios como generadores de identidad digital}

Los repositorios documentales han ido evolucionando desde su uso básico de sacar a la luz trabajos no publicados (preprints), a constituirse en una verdadera ventana para construir la identidad digital de autores e instituciones. Actualmente van más allá de las actividades de organizar, archivar, preservar documentos, y tienen un papel determinante como difusores de la producción intelectual de conocimiento.

Valga como ejemplo la descripción del repositorio Digital. CSIC, que indica que es una "estrategia de difusión de las pu- blicaciones científicas, ofrece la posibilidad de incrementar la visibilidad de las mismas y de llegar a un número mayor de lectores, lo que potencialmente se traducirá además en un número más elevado de citas a los trabajos".

http://digital.csic.es/faqs/\#faq1

Pero ¿qué es la identidad digital? Haciendo un símil, al igual que medimos el impacto medioambiental de nuestra sociedad sobre los recursos naturales con la "huella de carbono", en el mundo virtual empieza a definirse el concepto de "huella digital" (Deloitte, 2012) o activos digitales (Cantera, 2013) como el impacto de nuestra presencia en la Red, el rastro de información y conocimiento que generamos a lo largo de nuestra vida digital.

Las personas tenemos varias identidades digitales correspondientes a nuestras diferentes facetas (personal, profesional, consumidor, socio-cultural...). Deloitte (2012) afirma "que el número de credenciales que definen a un individuo ha crecido exponencialmente y la mayoría de usuarios cuentan con alguna forma de huella digital".

Nuestro entorno cambia y se expande a través de las diferentes redes a las que estamos conectados, por lo que gestionar las identidades resulta cada vez más complejo. Por ello se busca poder hacerlo mediante una única tecnología.

En el caso concreto de la documentación, Giones-Valls y Serrat-Brustenga (2010) indican que "la gestión de la identidad digital personal se define como la habilidad de gestionar con éxito la propia visibilidad, reputación y privacidad en la red como un componente inseparable y fundamental del conjunto de habilidades informacionales y digitales, las cuales se han convertido en fundamentales para vivir en la sociedad informacional".

Es de vital importancia el uso que damos a nuestros activos digitales (cuentas, perfiles, webs, blogs, listas, documentos en repositorios...) para tener una notoriedad correcta y una buena reputación en la Red. Debe controlarse con eficacia la identidad digital, ya que ésta ha superado las fronteras de nuestro PC y debemos ser conscientes de que la información que define quién somos, qué hacemos, dónde estamos, cuándo decidimos, por qué actuamos y cómo pensamos se encuentra disgregada y distribuida por la nube de internet.

Gartner (2012) asegura que "la nube reemplazará gradualmente al PC como el lugar donde las personas mantienen su contenido personal, tienen descritas sus preferencias, el acceso a sus servicios, convirtiéndose en el centro de su vida digital".

Aunque existen servicios que ayudan a la identificación y estandarización de los nombres de los autores (como Author Resolver, Inspire, IraLIS, Scopus Author ID, ResearcherID y 


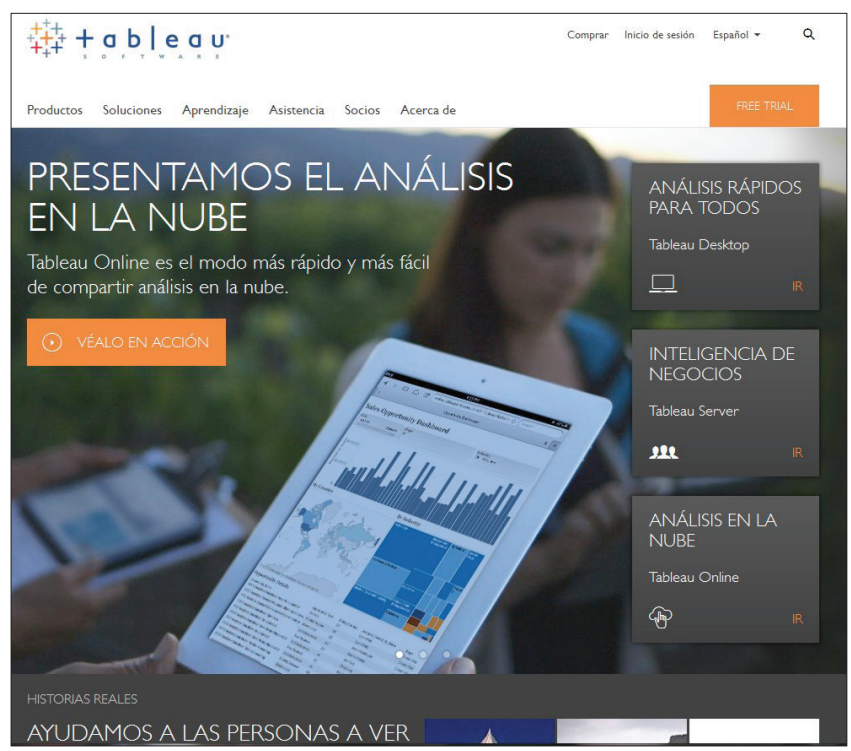

Figura 1. Tableau

http://www.tableausoftware.com/es-es

recientemente Orcid -que parece por fin el sistema definitivo-) carecen de una visión global de la identidad digital y aportan soluciones parciales, aunque todos tienden a vincular los nombres de los investigadores a sus instituciones y publicaciones, favoreciendo el proceso de descubrimiento científico.

\section{Repositorios, big data y open data}

Según IDC (Gantz et al., 2008) la producción digital total del mundo fue de 161 exabytes en 2006 y se preveía crecer hasta 988 exabytes en 2010, es decir, multiplicarse por 6 en cuatro años ( 1 exabyte $=1.000$ millones de gigabytes). Otra estimación de IDC-EMC (2011) prevé que el volumen de datos alcance los 35.000 exabytes en 2020 .

Un parte de estos datos corresponde a la información que manejamos usualmente los bibliotecarios y documentalistas, mucha de la cual se ubica en repositorios. Es difícil saber el número exacto de repositorios existentes, y mucho más conocer el número de documentos (textos, presentaciones, audios, vídeos...) que albergan. Algunos servicios pueden ofrecernos una idea:

- Ranking web de repositorios, del Laboratorio de Cibermetría del CSIC, tiene censados 1.650: http://repositories.webometrics.info/es

- Directory of Open Access Repositories (OpenDOAR) de la University of Nottingham lista 2.340: http://www.opendoar.org

- Registry of open access repositories (ROAR) de la University of Southampton tiene registrados 3.480: http://roar.eprints.org

Los sistemas con robots recolectores o cosechadores (harvesters) pueden también darnos una indicación del volumen de documentos accesibles:

- Recolecta, de la Fundación Española para la Ciencia y la Tecnología (Fecyt), indexa los registros bibliográficos de 286 colecciones y repositorios.

http://www.recolecta.net

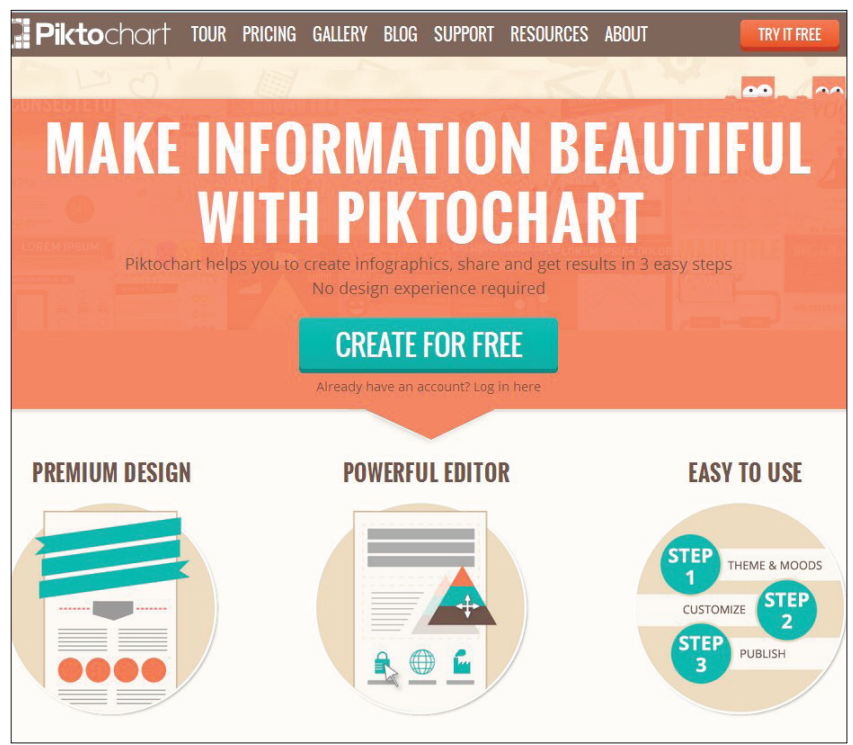

Figura 2. Piktochart

http://piktochart.com

- Hispana, del Ministerio de Educación, Cultura y Deporte, indexa 4,6 millones de documentos y otros objetos digitales procedentes de 202 repositorios.

http://hispana.mcu.es

- Universia indexa casi 18 millones de documentos, tesis, etc., de 226 repositorios.

http://biblioteca.universia.net

Por último citaremos OAlster, de OCLC, con 23 millones de registros recolectados de 1.100 repositorios.

http://www.oclc.org/oaister.en.html

Como en otros tipos de acumulaciones de datos e información, en los repositorios -todos los citados son libremente accesibles- es posible utilizar aplicaciones de inteligencia (business intelligence) con las cuales pueden extraerse informes sobre autores e instituciones: qué, cuánto, cómo y con quién investigan, impacto obtenido, etc. Entre las aplicaciones de inteligencia cabe citar desde las hojas de cálculo, hasta softwares como reporting and querying, online analytical processing (olap), cuadros de mando integrales (dashboards), minería de datos (data mining), etc. Todos estos sistemas han culminado en una de las grandes tendencias tecnológicas actuales: big data, que es el análisis masivo de ingentes cantidades de datos (logs de webs, datos meteorológicos, estelares, biológicos...) para extraer tendencias y conclusiones que sirvan para tomar decisiones. Las técnicas big data permiten tratar cantidades de información sin precedentes, para las cuales hasta ahora no existían ni software ni máquinas suficientemente rápidas.

\section{Big data}

Kusnetzky (2010) define las técnicas big data (grandes cantidades de datos) como las herramientas, procesos y procedimientos que permiten crear, manipular y gestionar conjuntos muy grandes de datos, así como sus instalaciones de almacenamiento. Según Dans (2011) big data es el "tratamiento y análisis de enormes repositorios de datos, tan desproporcionadamente grandes que resulta imposible tratarlos con las herramientas de bases de datos y analíticas convencionales". 


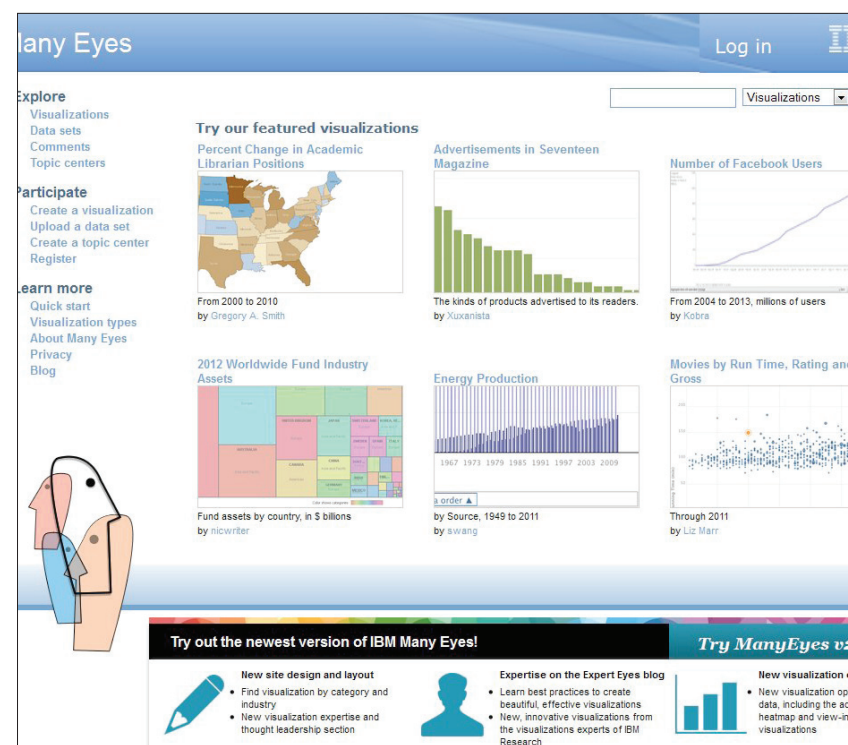

Figura 3. Many Eyes

http://www-958.ibm.com/software/data/cognos/manyeyes

Martínez-López (2012) afirma que tratar con volúmenes de información fuera de lo habitual se convierte en un problema de varios órdenes: de escalabilidad (¿podemos seguir creciendo?, ¿hasta cuánto?); de tratamiento (¿podremos tratar esos datos? ¿y cuándo tengamos el doble?); y de disponibilidad (¿es viable mantener online todos estos datos?).

La información contenida en la mayoría de repositorios documentales individuales se pueden analizar con las técnicas y herramientas clásicas, pero si se pudieran agregar todos ellos configurando una conjunto de datos (data set) distribuido, entonces se necesitarían técnicas big data.

Es pronto para saber cuál será la trascendencia y alcance futuro de los grandes conjuntos de datos, tanto en el ámbito profesional como para la ciudadanía en general, pero Tim Berners-Lee (TEDtalks, 2009) afirmó: "si la gente coloca sus datos en la Web, junto a datos gubernamentales, científicos, y los propios de la comunidad, podrán ser usados por otras personas en formas que nunca podrían haberse imaginado".

\section{Open data}

Son open data (datos abiertos o en abierto) los que se pueden capturar, tratar, analizar, modificar, reutilizar o difundir libremente para proporcionarles un nuevo valor informativo.

Mazzo-Iturriaga (2010) dice que "open data se asemeja al movimiento open source, pero a diferencia de éste no apunta a desarrollar software sino a posibilitar el acceso y reutilización de datos". Peset, Ferrer-Sapena y Subirats-Coll (2011) enfocan el concepto destacan la importancia de los estándares técnicos, de forma que para que los datos sean realmente abiertos y reutilizables deben ofrecerse en formatos normalizados. Estas autoras también se refieren a la exposición de los datos en la Web descritos en RDF (resource description framework), con indicación de la relación que puede existir entre esos datos y otros.

Si los repositorios de datos en abierto aplicaran estándares -y por tanto, gracias a esto, los datos fueran reutilizables-, permitirían generar clasificaciones, ontologías y formas de

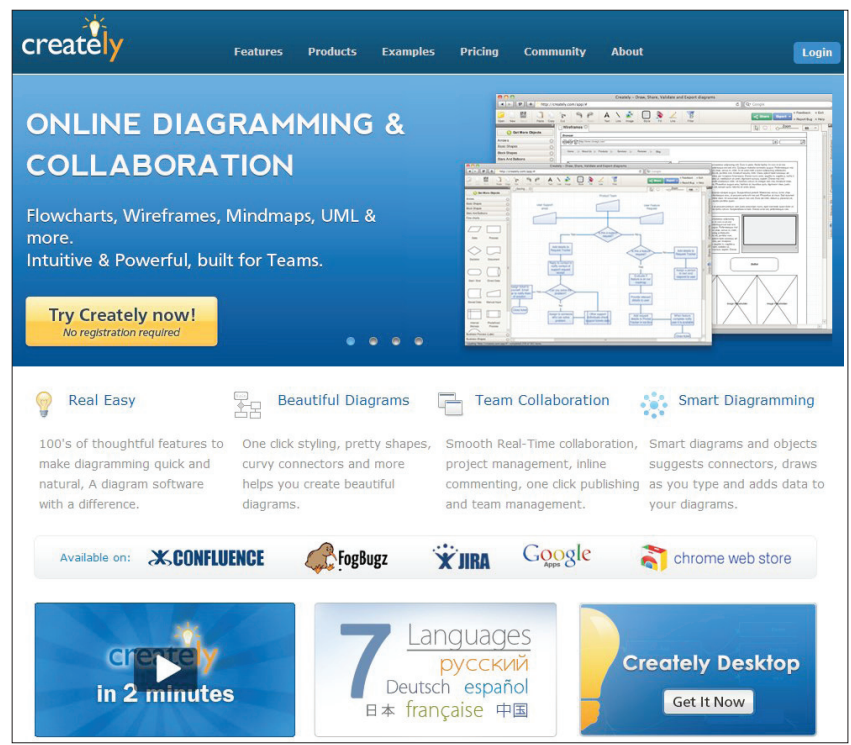

Figura 4. Creately http://creately.com

explotación nuevas mediante las técnicas de minería de datos, big data y visualización.

\section{Visualización de datos en repositorios}

La mayoría de repositorios documentales se han centrado en mejorar el almacenamiento, organización, clasificación, interoperabilidad, accesibilidad, usabilidad..., pero se han preocupado poco de aplicar técnicas de visualización de datos que harían la información más clara, atractiva y fácil de comprender. La presentación de la información de forma gráfica es una forma de análisis que permite identificar tendencias y patrones de relaciones que de otro modo serían muy difíciles de encontrar o ver.

Las técnicas de visualización de datos se encuentran muy ligadas a big data, pues son muy adecuadas para presentar los resultados resumidos del análisis de grandes cantidades de información. Algunos ejemplos de sistemas que permiten crear infografías online se citan en el blog BVA innovación (2013):

Tableau (figura 1)

http://www.tableausoftware.com/es-es

Piktochart (figura 2)

http://piktochart.com

Many Eyes (figura 3)

http://www-958.ibm.com/software/data/cognos/ manyeyes

Creately (figura 4)

http://creately.com

Visual.ly

http://visual.ly

Los sistemas de realización de infografías y de visualización de datos en general constituyen una parte de la tendencia conocida como narrativa digital (digital storytelling). A los repositorios, sean documentales o de datos, se les pueden incorporar sistemas de visualización que permitan consultarlos por diferentes caminos siguiendo las necesidades o deseos del usuario, en tiempo real (Shirky, 2012). Ferrer- 


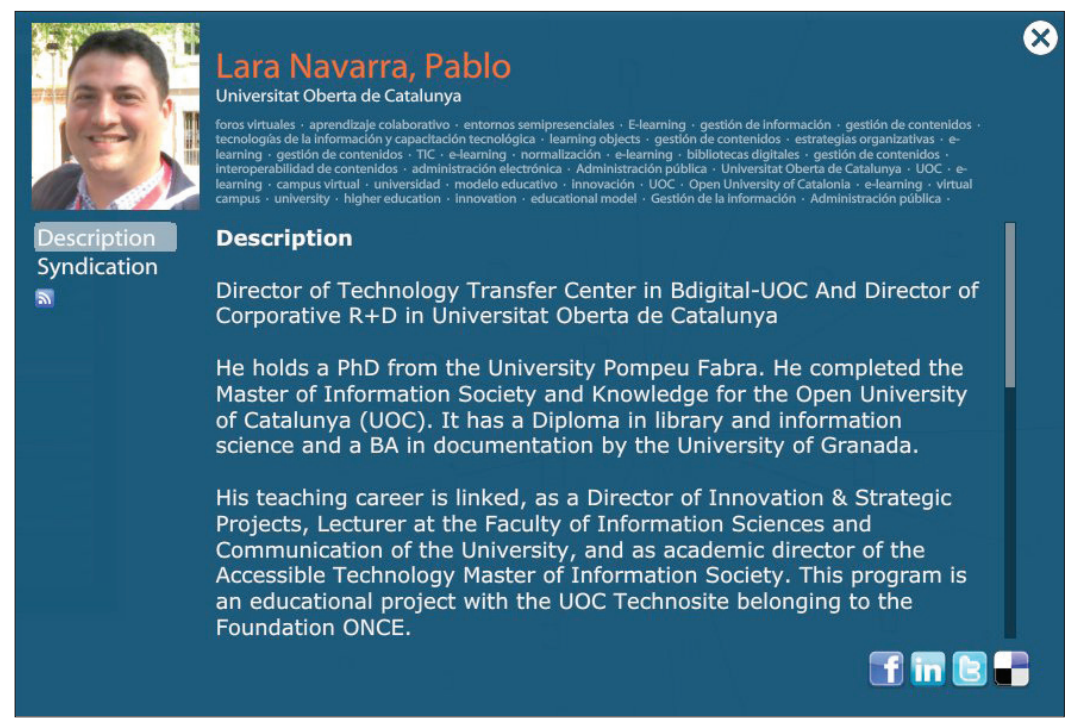

Figura 5. Modelo de ficha de autor y descripción

Sapena y Sánchez-Pérez (2013) apuntan que para contar buenas historias se necesitan capacidades para el análisis de los datos y buenas visualizaciones.

Según Díaz-Delgado (2009) la narrativa digital es "enfrentarse a un nuevo paradigma discursivo: el hipertexto, un sistema de escritura electrónica que organiza información de modo no lineal, con base en estructuras red, esto es, estructuras constituidas por nodos y enlaces". Y, desde un punto de vista documental, Herrera (2011) propone estrategias para desarrollar una taxonomía en narrativa digital, en la que prevalecen la capacidad de contar de una forma dinámica, con datos en tiempo real y una distribución masiva.

En el contexto de este artículo, consideramos importante la capacidad que deben tener los repositorios documentales de narrar visualmente estructuras de relaciones, como mí- nimo entre autores, documentos e instituciones.

\section{SocialNet: gestión de datos de repositorios documentales}

El proyecto SocialNet se concibió para incorporar las tendencias tecnológicas descritas en las secciones anteriores: identidad digital, big data, datos abiertos y visualización a los repositorios documentales. Aclaramos que en este momento SocialNet funciona sobre un repositorio de prueba con un número limitado de nodos por lo que no se trata propiamente de un ejemplo de big data, pero podría serlo aplicado a un conjunto mayor.

http://www.innovauoc.org/socialnet

\section{Identidad digital}

SocialNet presenta toda la información que genera un autor, tanto interna al repositorio como en internet, una vez deposita un documento. Se trabaja el concepto de huella documental, que aglutina la producción del mismo, su red de colaboradores, y sus redes personales y profesionales, para de esta forma obtener una visión global del mismo.

Para ello se genera una ficha que recoge todo su activo digital disponible, y se le asignan automáticamente una o más instituciones, y un conjunto de palabras clave extraídas de los documentos producidos tanto por el autor como por sus colaboradores. Estas etiquetas representan el conocimiento que tiene un autor, bien por él mismo o bien por sus coautores a quienes se supone puede preguntar. Es decir, se le otorga una nueva dimensión en su identidad digital, ya que, se le atribuye tanto el conocimiento real propio, como el potencial que puede obtener de su red de colaboradores. En la actualidad es tan importante identificar en la misma red "lo que se sabe" como "quién sabe lo que uno no sabe".

En la ficha de cada autor (figura 5) se creó la sección "syndication" con el listado de documentos con los que está relacionado. Este apartado permite acceder a cada documento haciendo clic sobre su título. Para acabar de definir esta identidad digital las fichas tienen una pequeña biografía y la vinculación con las redes sociales externas, lo que permite obtener una visión completa del autor y de sus conocimientos. 


\section{Big data y open data}

El problema a resolver no era tanto la capacidad del tratamiento masivo de información, sino las dificultades en la extracción de los datos en abierto y su homogeneidad. Como indica Abril-Martorell (2013), consejero delegado de Prisa, "lo más interesante del big data no es la cantidad de información a la que podremos acceder, sino en qué medida seremos capaces de utilizarla correctamente para aportar valor".

Para resolver esta cuestión, se analizaron y compararon diferentes estándares como Learning object metadata (LOM), Sharable content object reference mode (Scorm) y Dublin core cualificado $(D C)$. Sea decidió trabajar con $D C$ cualificado, y se creó un sistema de notación y etiquetado en XML $D C$ para marcar los campos de cada registro. Para ello se utilizó un cosechador (harvester) que confirmó la falta de estandarización de los datos que se encuentran de libre acceso en los repositorios documentales.

Una vez extraídos los datos, el trabajo se centró en poder tratarlos y visualizarlos en tiempo real.

Con el fin de poder visualizar conjuntos de datos complejos (por ej., cuando hay muchos coautores o muchos documentos) se podrían solapar líneas y nodos, se diseñaron funciones que permiten ajustar los parámetros básicos del sistema de visualización.

\section{Visualización de información}

El sistema permite generar visualizaciones complejas de datos de repositorios documentales. Los usuarios pueden narrarse su propia historia navegando por los autores y documentos de forma interactiva.

Al introducir un nombre de autor la cosechadora rastrea en los repositorios agregados, y una vez hallados los trabajos se vuelcan en la base de datos. Seguidamente se extraen los coautores de los que a su vez se buscan los trabajos. De esta manera, la red del sistema se va retroalimentando y crece paulatinamente a medida que se introducen nuevos documentos.

El componente principal del sistema es un visor de grafos, un espacio hiperbólico que se autoordena y dimensiona para que los elementos representados no se solapen, en el cual aparecen dos tipos de nodos: autor y documento, así como las relaciones entre ellos (figura 6).

Los nodos de autor tienen un círculo con un grosor de línea proporcional a la cantidad de documentos que ha escrito. Los nodos de documento se representan por un símbolo con un grosor de borde proporcional a la cantidad de autores con que están relacionados. Los colores indican la procedencia del documento. Al abrir un nodo se despliega el conjunto de conexiones con los que está relacionado y se dibujan automáticamente las relaciones con otros nodos en la red.

Al pasar el ratón sobre un nodo aparecen iconos adicionales que permiten esconder el nodo al visor, o en el caso de los autores, acceder a la ficha de información detallada. También aparece una ventana flotante con una información mínima sobre cada nodo, que ayuda a reconocerlo.

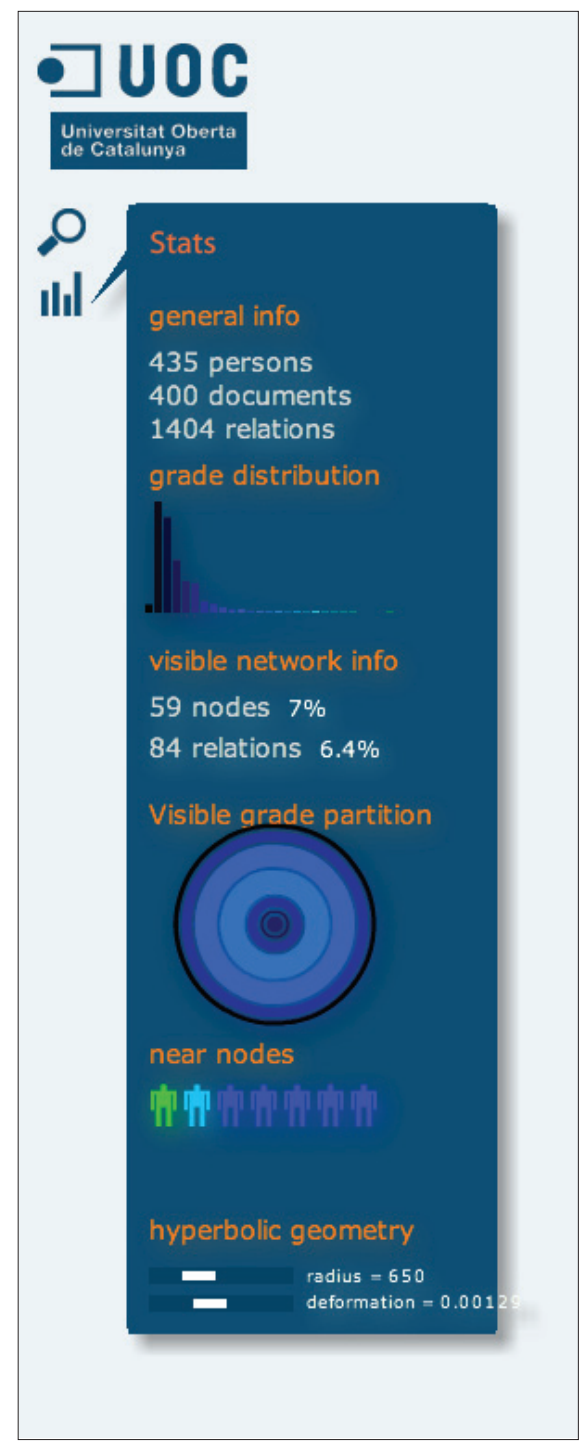

Figura 7. Panel de indicadores de red

Estadísticas y detalles de la subred de autores visualizada en la figura 6. El panel (que aparece haciendo clic en el pequeño icono en forma de diagrama de barras) contiene las siguientes secciones:

a) General info: Número de autores, documentos y relaciones de la totalidad de la red tratada.

b) Grade distribution: diagrama de barras representando la distribución de autores según el grado de relaciones (primer coautor, coautor del coautor, etc.).

c) Visible network info: número y proporción de nodos, y relaciones visibles en pantalla.

d) Visible grade partition: Gráfica radial con el no de nodos (autores) según el grado o distancia al autor.

e) Near nodes: listado de nodos más próximos.

f) Hyperbolic geometry: controles del radio de la representación visual y de deformación de la geometría para centrar la parte que se desea visualizar.

\section{Disponibilidad del producto}

La investigación y el desarrollo realizados en torno al proyecto SocialNet para el tratamiento de datos de repositorios de información y visualización de relaciones autores-documentos, nos permiten abordar nuevos modelos de explotación de los repositorios, tanto para generadores como para distribuidores de información. Así, por ejemplo, SocialNet ayudó a la empresa Bestiario a diseñar usos de su producto 
Quadrigram, una herramienta de gestión de grandes datos y datos abiertos, y diseño de narraciones visuales.

SocialNet se diseñó como un modelo ad hoc para estudiar y analizar la generación de redes sociales en unos repositorios concretos, pero podría aplicarse a otros como E-LIS o Dialnet, por ejemplo, y de esta manera ofrecer nuevos modelos de visualización de las redes de información subyacentes en esos repositorios.

Sin embargo, hacer una adaptación o un nuevo diseño a partir de la estructura básica de SocialNet saldría muy caro, pues el modelo descrito en este artículo estaba dirigido a una explotación muy concreta. Sería recomendable acudir a la citada empresa Bestiario, desarrolladora de Quadrigram, producto que puede ser utilizado por cualquier repositorio que iniciase un nuevo proyecto sobre sus datos. Quadrigram se ofrece con unas tarifas bastantes asequibles, como se describe en su web, y también cuenta con asesoramiento especializado de sus desarrolladores para orientar las posibilidades y alcance de un proyecto.

\section{Conclusiones}

Se vislumbra un gran recorrido para los profesionales de la información tanto en el campo académico como en el profesional y empresarial, con diferentes especialidades y campos de conocimiento emergentes, pero para ello hay que estar atento a la evolución de las tecnologías como por ejemplo visualización de datos, identidad digital, datos abiertos, grandes conjuntos de datos, y otras que a buen seguro aparecerán.

Los bibliotecarios-documentalistas actuales están familiarizados con las bases de datos y los repositorios documentales, pero deberían pensar en dotarlas de valor añadido mediante sistemas de narrativa digital: infografías y sistemas de visualización de datos para que las consultas por parte de los usuarios finales resulten más eficaces, y sea más fácil sacar conclusiones. De esta forma a partir de los conjuntos de datos se extrae información, que a su vez deriva en conocimiento colectivo.

SocialNet es un ejemplo de cómo se pueden interconectar los datos para dotarlos de un nuevo valor informativo. Destaca su capacidad de organizar visualmente una red de coautoría, de ofrecer información estadística tanto individual como colectiva, ayuda a verificar el conocimiento que tiene un autor, sus campos de conocimiento y los de sus pares.

\section{Bibliografía}

Abril-Martorell, Fernando (2013). Grandes datos, grandes historias, julio.

http://toyoutome.es/blog/grandes-datos-grandeshistorias $/ 22218$
Accenture (2012). Accenture technology vision. http://www.accenture.com/es-es/Pages/insight-accenturetechnology-vision-2012.aspx

BBVA Innovación (2013). Innovadata: Periodismo de datos e innovación, mayo.

https://www.centrodeinnovacionbbva.com/blogs/planta29/ posts/22047-innovadata-periodismo-de-datos-e-innovacion

Berners-Lee, Tim (2009). "The next Web of open, linked data". TED, February.

https://www.ted.com/talks/tim_berners_lee_on_the_next_ web.html

http://www.w3.org/Designlssues/LinkedData.html

Cantera, José (2013). Descodificación del ADN de la experiencia de cliente del ecommerce en España.

http://www.kpmgdigital.es/?p=701

Dans, Enrique (2011). “Big Data: una pequeña introducción". El blog de Enrique Dans, 19 octubre.

http://www.enriquedans.com/2011/10/big-data-unapequena-introduccion.html

Deloitte (2012). Predicciones de Deloitte para el sector de tecnología, medios de comunicación y telecomunicaciones http://www.deloitte.com/assets/Dcom-Mexico/Local\%20 Assets/Documents/mx(es-mx)TMT2012_Esp.pdf

Díaz-Delgado, Natalia (2009). Narrativa digital. http://narrativa--digital.blogspot.com.es

Ferrer-Sapena, Antonia; Sánchez-Pérez, Enrique A. (2013). “Open data, big data: ¿hacia dónde nos dirigimos?". Anuario ThinkEPI, v. 7, pp. 150-157.

Gantz, John F. et al. (2008). The diverse and exploding digital universe. An updated forecast of worldwide information growth through 2011. An IDC white paper, sponsored by EMC, 16 pp., March 2008.

http://www.emc.com/collateral/analyst-reports/diverse- 
exploding-digital-universe.pdf

Gartner (2013). Gartner identifies the top 10 strategic technology trends for 2013.

http://www.gartner.com/newsroom/id/2209615

Giones-Valls, Aina; Serrat-Brustenga, Marta (2010). “La gestión de la identidad digital: una nueva habilidad informacional y digital". BiD: textos universitaris de biblioteconomia i documentació, juny, n. 24.

http://bid.ub.edu/24/giones2.htm

Herrera, Raquel (2011). "Propuesta de estrategias para desarrollar una taxonomía en narrativa digital". Hipertext.net, n. 9. http://www.upf.edu/hipertextnet/numero-9/taxonomianarrativa-digital.html

IDC-EMC (2011). Digital universe study. Extracting value from chaos. http://www.emc.com/leadership/programs/digital-universe.htm

KPMG (2000). Knowledge management research report. http://www.providersedge.com/docs/km_articles/kpmg_ km_research_report_2000.pdf
Kusnetzky, Dan (2010). "What is “Big Data”?". ZDNet, 16 febrero. http://www.zdnet.com/blog/virtualization/what-is-bigdata/1708

Martínez-López, Héctor (2012). "The next big thing”. El blog d'UPCnet, 27 enero.

http://blog.upcnet.es/the-next-big-thing

Mazzo-Iturriaga, Rodrigo (2010). Linked open data: qué es y ejemplos en el mundo

http://www.bcn.cl/de-que-se-habla/open-data-link-data

Peset, Fernanda; Ferrer-Sapena, Antonia; Subirats-Coll, Imma (2011). "Open data y linked open data: su impacto en el área de bibliotecas y documentación". El profesional de la información, marzo-abril, v. 20, n. 2, pp. 165-173.

http://dx.doi.org/10.3145/epi.2011.mar.06

Shirky, Clay (2012). “Cooperation and corruption". Crooked Timber. http://crookedtimber.org/2012/07/02/cooperation-andcorruption

\section{Directorio EXIT \\ (EXpertos en Tratamiento de la Información) http://directorioexit.info}

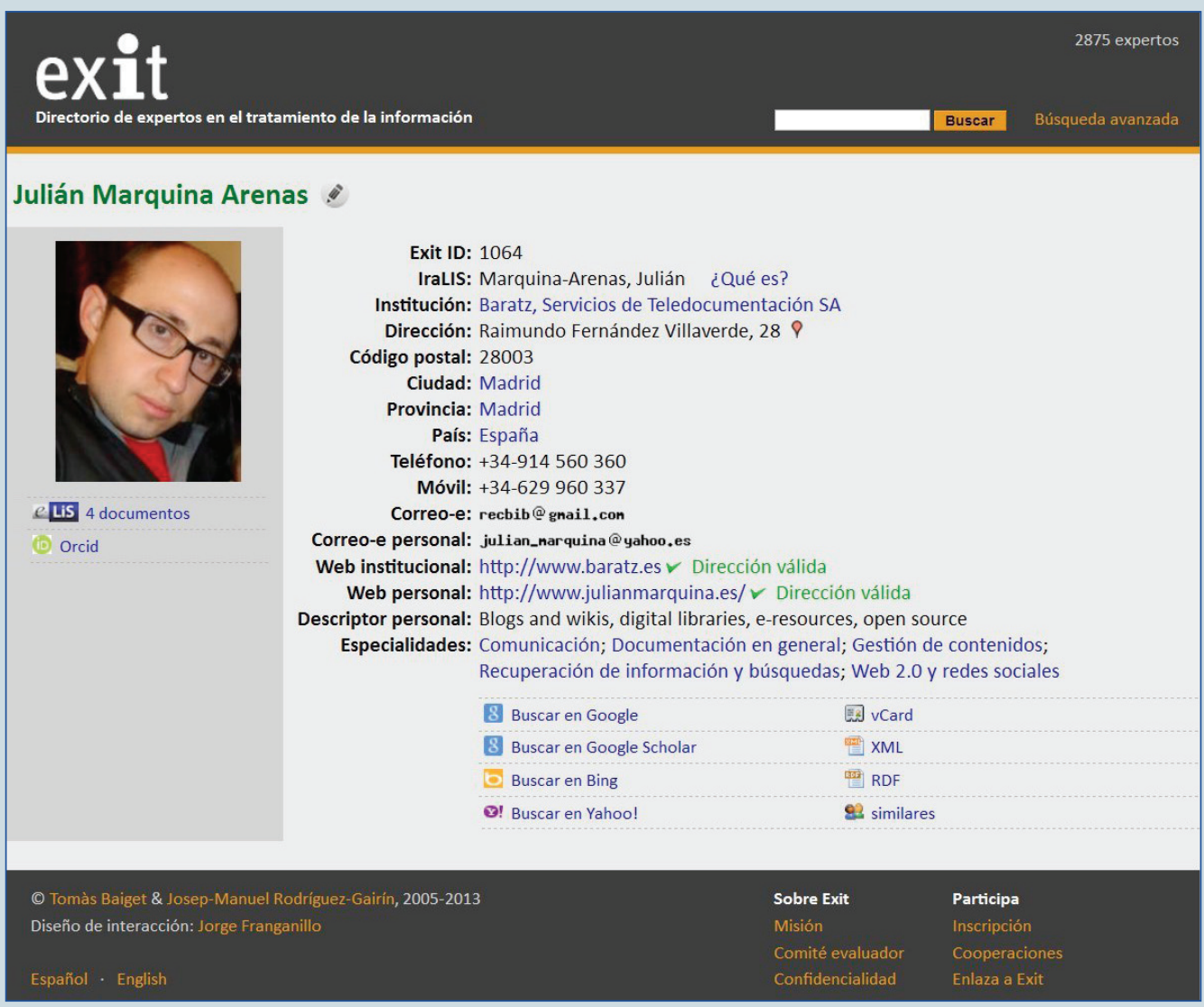

\section{Una ventana a las oportunidades profesionales}

La nueva versión 2013 localiza la dirección postal en Google maps y permite buscar perfiles de expertos similares a uno dado. 